\title{
Consumption of differently processed milk products in infancy and early childhood and the risk of islet autoimmunity
}

\author{
Katariina Koivusaari ${ }^{1,2} \dagger$, Essi Syrjälä ${ }^{3} \dagger$, Sari Niinistö ${ }^{1}$, Hanna-Mari Takkinen ${ }^{1,3}$, Suvi Ahonen ${ }^{1,3,4}$,
} Mari Åkerlund $^{1,3}$, Tuuli E. Korhonen ${ }^{1}$, Jorma Toppari ${ }^{5,6}$, Jorma Ilonen ${ }^{7}$, Jaakko Peltonen ${ }^{8}$, Jaakko Nevalainen ${ }^{3}$, Mikael Knip ${ }^{9,10,11,12}$, Tapani Alatossava ${ }^{2}$, Riitta Veijola ${ }^{13}$ and Suvi M. Virtanen ${ }^{1,3,4,11 *}$ ${ }^{1}$ Department of Public Health Solutions, Finnish Institute for Health and Welfare, FI-O0271 Helsinki, Finland

${ }^{2}$ Department of Food and Nutrition, University of Helsinki, FI-OOO14 Helsinki, Finland

${ }^{3}$ Unit of Health Sciences, Faculty of Social Sciences, Tampere University, FI-33014 Tampere, Finland

${ }^{4}$ Tays Research, Development and Innovation Center, Tampere University Hospital, FI-33521 Tampere, Finland

${ }^{5}$ Department of Pediatrics, Turku University Hospital, FI-20521 Turku, Finland

${ }^{6}$ Research Centre for Integrative Physiology and Pharmacology, Institute of Biomedicine, University of Turku, FI-20520 Turku, Finland

${ }^{7}$ Immunogenetics Laboratory, Institute of Biomedicine, University of Turku and Clinical Microbiology, Turku University Hospital, FI-20520 Turku, Finland

${ }^{8}$ Faculty of Information Technology and Communication Sciences, Tampere University, FI-33100 Tampere, Finland

${ }^{9}$ Children's Hospital, University of Helsinki and Helsinki University Central Hospital, FI-O0281 Helsinki, Finland

${ }^{10}$ Pediatric Research Center, Research Program for Clinical and Molecular Medicine, University of Helsinki, FI-OOO14 Helsinki, Finland

${ }^{11}$ Tampere Center for Child Health Research, Tampere University Hospital, FI-33014 Tampere, Finland

${ }^{12}$ Folkhälsan Research Center, FI-OO29O Helsinki, Finland

${ }^{13}$ Department of Pediatrics, PEDEGO Research Unit, Medical Research Center Oulu, University of Oulu and Oulu University Hospital, FI-90014 Oulu, Finland

(Submitted 14 November 2019 - Final revision received 31 January 2020 - Accepted 11 February 2020 - First published online 27 February 2020)

\section{Abstract}

Several prospective studies have shown an association between cows' milk consumption and the risk of islet autoimmunity and/or type 1 diabetes. We wanted to study whether processing of milk plays a role. A population-based birth cohort of 6081 children with HLA-DQB1conferred risk to type 1 diabetes was followed until the age of 15 years. We included 5545 children in the analyses. Food records were completed at the ages of 3 and 6 months and 1, 2, 3, 4 and 6 years, and diabetes-associated autoantibodies were measured at 3-12-month intervals. For milk products in the food composition database, we used conventional and processing-based classifications. We analysed the data using a joint model for longitudinal and time-to-event data. By the age of 6 years, islet autoimmunity developed in 246 children. Consumption of all cows' milk products together (energy-adjusted hazard ratio 1.06; $95 \%$ CI 1.02, 1.11; $P=0.003$ ), non-fermented milk products (1.06; $95 \%$ CI 1.01, 1.10; $P=0.011)$ and fermented milk products $(1.35 ; 95 \%$ CI $1 \cdot 10,1 \cdot 67 ; P=0.005)$ was associated with an increased risk of islet autoimmunity. The early milk consumption was not associated with the risk beyond 6 years. We observed no clear differences based on milk homogenisation and heat treatment. Our results are consistent with the previous studies, which indicate that high milk consumption may cause islet autoimmunity in children at increased genetic risk. The study did not identify any specific type of milk processing that would clearly stand out as a sole risk factor apart from other milk products.

Key words: Children: Milk products: Islet autoimmunity: Homogenisation: Heat treatment: Joint models: Survival analysis

The incidence of type 1 diabetes increased in recent decades across Europe, although it plateaued after 2005 in Finland, the incidence rate being 60.9 per 100000 person-years in 2009-2013 ${ }^{(1)}$. Several prospective studies have shown an association between high consumption of cows' milk products and an increased risk of islet autoimmunity and/or type 1 diabetes $^{(2-6)}$, but the findings regarding early cows' milk introduction are contrasting ${ }^{(7,8)}$.

Abbreviations: DIPP, Type 1 Diabetes Prediction and Prevention; IQR, interquartile range.

* Corresponding author: Suvi M. Virtanen, email suvi.virtanen@thl.fi

$\dagger$ These authors contributed equally to this work. 
It is unclear which factor(s) in milk, if any, is associated with the risk of type 1 diabetes. The suspected factors include bovine insulin ${ }^{(9)}$, milk fat and milk protein ${ }^{(4)}$. The effect of protein hydrolysation of infant formula has been studied previously ${ }^{(10,11)}$. Although a strong heat treatment of milk can destroy the biochemical activity of bovine insulin ${ }^{(12)}$, alter the stability of protein structures in milk $^{(13)}$ and change the immune response to milk in vitro ${ }^{(14)}$, the effect of processing in relation to the development of type 1 diabetes has not been studied.

The aim of the present study was to clarify the association between consumption of differently processed milk products and the risk of islet autoimmunity by using a Finnish birth cohort data. Using food records allowed us to use a novel homogenisation and heat treatment-based classification for milk products. In Finland, milk consumption per capita is among the highest in the world and the selection of milk products on the market is wide.

\section{Methods}

\section{Participants}

Participants of the present study ( $n$ 6081) were born between September 1996 and September 2004 in Oulu and Tampere University Hospitals in Finland. The Type 1 Diabetes Prediction and Prevention (DIPP) Nutrition Study is a part of the DIPP Study which is a prospective, population-based birth cohort of Finnish children with a high or moderate genetic risk for type 1 diabetes. The newborn infants' genotypes for specific HLA-DQB1 alleles were determined from cord blood. The high-risk genotype was defined as HLA-DQB1*02/*03:02 and the moderate-risk genotypes as HLA-DQB1*03:02/x, with $\mathrm{x}$ not equal to $* 22, * 03: 01$ or $* 06: 02^{(15)}$. Families with a newborn infant carrying HLA-conferred susceptibility to type 1 diabetes were invited to participate in the study. During the follow-up, growth and antibodies were followed at the ages of 3, 6, 9, 12, 18 and 24 months and then annually. If antibodies were detected, the participants were followed every 3 months. The blood samples for autoantibody monitoring were collected by venepuncture up to the age of 15 years.

We included 5545 children in the analyses: the inclusion criterion was having at least one 3-d food record and at least one autoantibody measurement after the food record. Among these 5545 children, the median (interquartile range (IQR)) number of autoantibody measurements was 14 (IQR 6-18) and 3-d food records 5 (IQR 3-8) per child. Of the children, 5506 were eligible for the energy-adjusted analyses and 5400 for the breast milk analyses (online Supplementary Fig. 1). Information on child's sex and first-degree familial diabetes (any kind) was registered by a structured questionnaire completed by the parents after delivery.

\section{Ethics}

Parents gave their written informed consent for genetic testing of their newborn infant from the cord blood sample and for participation in the follow-up. The study adheres to the Declaration of Helsinki, and the local ethics committees have approved the study protocol.

\section{Dietary assessment}

The child's diet was assessed at the age of 3 and 6 months and 1 , $2,3,4$ and 6 years by a 3 -d food record. Collecting and processing of food consumption data have been described in detail earlier $^{(4)}$. The amount of breast milk was calculated based on children's growth and intake of other foods ${ }^{(16)}$. If the child was exclusively breastfed, the calculation was done based on the growth only. Trained research nutritionists entered the food record data using the Finnish National Food Composition Database Fineli and the in-house dietary calculation software Finessi, the National Institute for Health and Welfare. The food composition database and the connected dietary calculation software enabled the calculation of the food intake on an ingredient level ${ }^{(4)}$. Thus, it was possible, for example, to summarise the total intake of milk from different sources (plain milk and milk from dishes containing milk). The food consumption data were used up to the detection of islet autoimmunity or to the age at the last autoantibody measurement.

\section{Milk product classification}

As the conventional grouping for milk products, we used the following: cows' milk products, which consist of the following three classes: (i) non-fermented milk products which include milks, creams, ice creams, milk-based infant formulas and powder-like milk containing preparations; (ii) fermented milk products consisting of fermented milks and sour creams; and (iii) cheeses, including all kinds of cheeses from fresh cheeses to long-ripened cheeses. Infant formulas were categorised into two categories: (i) milk-based formulas including partially hydrolysed formulas; and (ii) extensively hydrolysed infant formulas.

In the novel processing-based classification, each milk product of the food composition database was classified into one of the following groups based on the homogenisation information: (1) homogenised, (2) non-homogenised or (3) fat-free; and into one of the following groups based on the heat treatment information: (1) low-pasteurised (typically $15 \mathrm{~s}$ at $73^{\circ} \mathrm{C}$ or corresponding conditions where milk alkaline phosphatase is inactivated) or less heat-treated, (2) high-pasteurised at $<100^{\circ} \mathrm{C}$ or (3) high-pasteurised at $\geq 100^{\circ} \mathrm{C}$ or sterilised ${ }^{(17)}$. Products that included fat not more than $0.5 \mathrm{~g} / 100 \mathrm{~g}$ or $0.5 \mathrm{ml} / 100 \mathrm{ml}$ were classified as fat-free. Details of the classification and consumption of different milk products have been described previously ${ }^{(17)}$. As an example, standard milks were classified as homogenised or fat-free and low-pasteurised, organic milks as non-homogenised or fat-free and low-pasteurised, and special milks (e.g. lactose-free milks) as homogenised or fat-free and high-pasteurised at $\geq 100^{\circ} \mathrm{C}$ or sterilised. Infant formulas were classified as homogenised and high-pasteurised at $\geq 100^{\circ}$ $\mathrm{C}$ or sterilised, yogurts as homogenised or fat-free and highpasteurised at $<100^{\circ} \mathrm{C}$, and most ripened cheeses as nonhomogenised and low-pasteurised.

\section{Definition of outcome}

As the outcome, we used islet autoimmunity. Islet cell antibodies were used as the primary testing for islet autoimmunity. If the child tested positive for islet cell antibodies, all the samples since 
birth were analysed for insulin autoantibodies, antibodies to the $65 \mathrm{kDa}$ isoform of glutamic acid decarboxylase and the tyrosine phosphatase-related islet antigen 2 molecule. The quantification of the antibody concentrations has been described previously ${ }^{(18)}$. Islet autoimmunity outcome was defined as repeated positivity for islet cell antibodies and one or more of the three biochemical antibodies analysed ${ }^{(19)}$. This outcome also includes children who developed type 1 diabetes before the islet autoimmunity outcome ( $n 34$ until the age of 6 years, $n 43$ until the age of 15 years). Data of type 1 diabetes diagnosis were obtained from the national paediatric diabetes registry.

\section{Statistical methods}

We used a joint model for longitudinal and time-to-event data with a current value association structure ${ }^{(20)}$ to analyse the association between consumption of different milk products or amount of breast milk and the risk of islet autoimmunity. A joint model consisted of two submodels fitted simultaneously: a linear mixed effects model for the milk product consumption measurements and a relative risk model for the islet autoimmunity risk. The linear mixed effects model determined the unobserved milk product consumption trajectories for the whole time period for every child, and the covariate trajectories were used in the relative risk model. The trajectory could be estimated, although some of the food records were missing. Use of the whole data in the model reduced the bias related to missing data as all children were included in the analyses and the number of the individual food records reflected the precision of the estimated trajectory and in the final standard errors of the estimates. The trajectories were used in the relative risk model with and without confounding factors until the end point for the particular child occurred.

We used flexible piecewise cubic polynomial spline functions in the linear mixed effects submodels. At times where consumption was no longer observed, for infant formulas beyond 3 years and for breast milk beyond 2 years, we used values drawn from a zero mean normal distribution with negligible variance to maintain the trajectory at zero and to improve model convergence. For breast milk, we additionally classified the children into breastfed and not breastfed and added this factor including the interactions between the spline basis functions to the linear mixed effects models to obtain better fit of the individual breast milk trajectories among those with no breast-feeding. Formulation for the breast milk trajectories is presented in the online Supplementary Methods. In the relative risk submodels, time-to-event data were used up to the age of 6 years, which was the last point of milk product consumption measurements. The end points that occurred after the age of 6 years were treated as right-censored at 6 years. Times-to-event for children with islet autoimmunity were set to the middle of the time interval between the last islet autoimmunity-negative and the islet autoimmunity-positive measurement. Otherwise, data were right-censored at the age of the last autoantibody measurement. Detailed information on the joint models used has been described previously ${ }^{(21)}$.

We adjusted the joint models for sex (boy or girl), HLAgenotype (high or moderate risk) and diabetes of the first-degree relative (yes, no or missing value), as these variables have been previously found to be potential confounders ${ }^{(21)}$. Missing values in diabetes of the first-degree relative were coded as a third level of the factor to enable the inclusion of those children in the model. The energy intake adjustment was done by the density approach $^{(22)}$ by dividing milk product consumption by total energy intake (MJ) in every measurement point, and by using this variable instead of the original absolute consumption in the models. As milk products were consumed in large amounts, hazard ratios were scaled to represent relative risk due to a $20 \mathrm{~g} / \mathrm{MJ}$ change in the energy-adjusted consumption.

To investigate whether the association between consumption of different milk products or amount of breast milk and the risk of islet autoimmunity remains after 6 years of age, until the age of 15 years, we used Cox regression models with the cumulative consumption of the different milk products as a time-independent covariate and time origin set at 6 years. Cumulative consumption was calculated as the area under the curve based on the individual milk product consumption estimates from the linear mixed effects model. Data for islet autoimmunity-negative children were rightcensored at the age of the last autoantibody measurement. Children who experienced the end point by 6 years of age were excluded from these analyses.

We could not use the joint model for extensively hydrolysed infant formulas, as they were consumed in small volume and only by 249 children. For studying the association between the consumption of extensively hydrolysed infant formulas and the risk of islet autoimmunity, we dichotomised the variable based on the information whether the child had consumed hydrolysed infant formulas (at least in one measurement point) or not. The association between dichotomised hydrolysed infant formulas and the risk of islet autoimmunity was analysed with the Cox regression model.

Analyses were implemented with R version 3.4.3 (R Foundation for Statistical Computing) by using coxph function from the survival package and jointModel function from the JM package version $1.4-8^{(23)}$.

\section{Results}

By the age of 6 years, islet autoimmunity developed in 246 children (4.4\%) at median age of $2 \cdot 5$ (IQR 1.3-3.6) years. In total, by the age of 15 years, islet autoimmunity developed in 348 children $(6.3 \%)$ with median age of 3.5 (IQR 1.8-6.6) years. The median follow-up time was $6 \cdot 0$ (IQR 3.1-6.0) years for the 6-year data and 10.0 (IQR 3.1-14.9) for the 15-year data. The dropout rates at 1, 2, 6 and 15-year follow-up were 8, 16, 32 and $67 \%$, respectively. Characteristics of the children are presented in Table 1 . The children consumed on an average slightly less than half a kg of cows' milk products per d (Table 1). At all ages, non-fermented milk products were consumed in larger amounts than fermented milk products (Fig. 1).

Every $100 \mathrm{~g}$ increment in consumption of cows' milk products was associated with a $7 \%$ increase in the risk of islet autoimmunity (Table 2). Both the higher consumption of non-fermented and fermented milk products were associated with a higher risk of islet autoimmunity according to the unadjusted and 
Table 1. Characteristics of the participating children

(Numbers and percentages; mean values and standard deviations)

\begin{tabular}{|c|c|c|c|c|c|c|c|c|c|c|}
\hline & \multicolumn{2}{|c|}{$\begin{array}{c}\text { Distribution of } \\
\text { children }(n 5545)\end{array}$} & \multicolumn{2}{|c|}{$\begin{array}{c}\text { Children } \\
\text { with islet } \\
\text { autoimmunity } \\
\text { by } 6 \text { years } \\
(n 246)\end{array}$} & \multicolumn{2}{|c|}{$\begin{array}{c}\text { Children } \\
\text { with islet } \\
\text { autoimmunity } \\
\text { by } 15 \text { years } \\
(n 348)\end{array}$} & \multicolumn{2}{|c|}{$\begin{array}{c}\text { Consumption } \\
\text { of cows' milk } \\
\text { products at } \\
\text { the age of } \\
1 \text { year }(\mathrm{g} / \mathrm{d})^{\star}\end{array}$} & \multicolumn{2}{|c|}{$\begin{array}{c}\text { Consumption } \\
\text { of cows' milk } \\
\text { products at } \\
\text { the age of } \\
3 \text { years }(\mathrm{g} / \mathrm{d}) \dagger\end{array}$} \\
\hline & $n$ & $\%$ & $n$ & $\%$ & $n$ & $\%$ & Mean & SD & Mean & SD \\
\hline \multicolumn{11}{|l|}{ Sex } \\
\hline Boys & 2950 & 53.2 & 148 & $5 \cdot 0$ & 212 & $7 \cdot 2$ & 572 & 312 & 559 & 286 \\
\hline Girls & 2595 & $46 \cdot 8$ & 98 & 3.8 & 136 & $5 \cdot 2$ & 567 & 292 & 542 & 267 \\
\hline \multicolumn{11}{|l|}{ HLA-DQB1-conferred risk } \\
\hline High $\left(\mathrm{DQB} 1{ }^{*} 02 /{ }^{*} 03: 02\right)$ & 1088 & 19.6 & 78 & $7 \cdot 2$ & 120 & 11.0 & 588 & 295 & 542 & 266 \\
\hline Moderate $\left(\mathrm{DQB} 1^{*} 03: 02 / \mathrm{x}\right) \ddagger$ & 4457 & $80 \cdot 4$ & 168 & $3 \cdot 8$ & 228 & 5.1 & 565 & 304 & 553 & 280 \\
\hline \multicolumn{11}{|l|}{ Familial diabetes } \\
\hline Yes & 329 & 5.9 & 30 & 9.1 & 41 & 12.5 & 563 & 322 & 536 & 277 \\
\hline No & 5001 & $90 \cdot 2$ & 210 & 4.2 & 299 & 6.0 & 568 & 302 & 551 & 276 \\
\hline Missing information & 215 & 3.9 & 6 & $2 \cdot 8$ & 8 & 3.7 & 615 & 273 & 576 & 327 \\
\hline
\end{tabular}

* $n$ of food records $=12827$.

$\dagger n$ of food records $=9766$.

$\ddagger x$ not equal to ${ }^{*} 02,{ }^{*} 03: 01$ or ${ }^{*} 06: 02$.

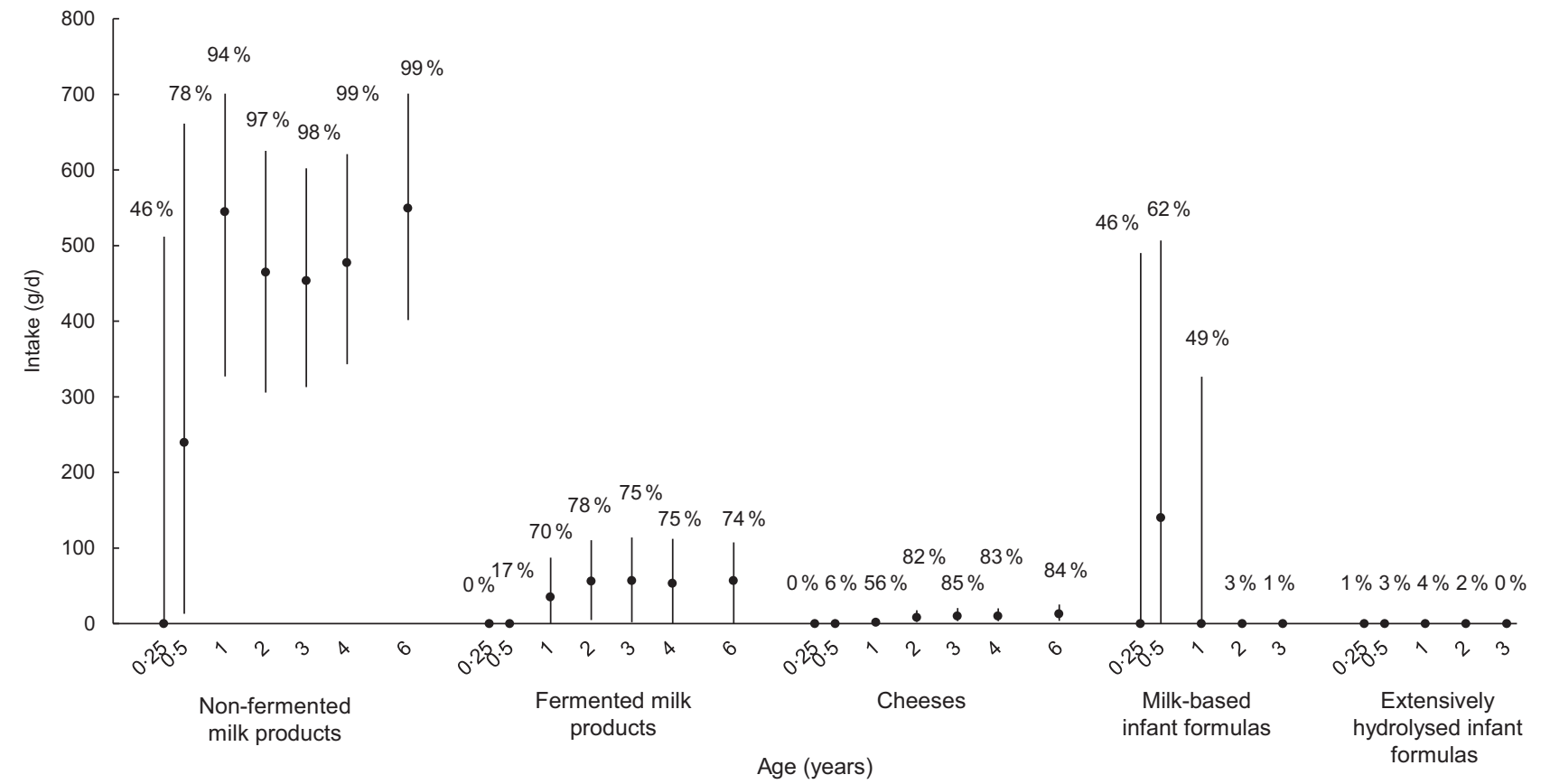

Fig. 1. Consumption of different cows' milk products at the age of 3 months ( $n 5175), 6$ months ( $n 4838), 1$ year ( $n 4333), 2$ years $(n 3555)$, 3 years $(n 3330), 4$ years ( $n$ 2979) and 6 years ( $n$ 2267) using conventional classification. Values are medians, with interquartile ranges represented by vertical bars. Values are calculated based on all the available data for the 5545 children included in the analyses. The percentages above the lines present the proportion of children using the particular milk product at that age point.

energy-adjusted models. We did not observe the association between the consumption of cheeses or cows' milk-based infant formulas and the risk of islet autoimmunity. The consumption of extensively hydrolysed infant formulas was not associated with the risk (hazard ratio $0.82 ; 95 \% \mathrm{CI} 0.44,1.56 ; P=0.558$ ) according to the Cox regression model.

Consumption of homogenised milk products was notably higher than the consumption of non-homogenised milk products at all age points after 3 months and consumption of fat-free milk products increased by age (Fig. 2). Strongly heat-treated milk products were consumed more than low-pasteurised ones during the first year of life, after which low-pasteurised milk products were consumed more (Fig. 2). The consumption of homogenised milk products was directly associated with the risk of islet autoimmunity (Table 2), whereas the consumption of non-homogenised or fat-free milk products was not associated with the risk. We first analysed separately the two strongest heat treatment classes, high-pasteurised and sterilised milk products, but since the hazard ratios were similar, we combined these two classes. A higher consumption of those milk products was associated with a higher risk of islet autoimmunity in the unadjusted model but not in the adjusted models (Table 2). 
(a)

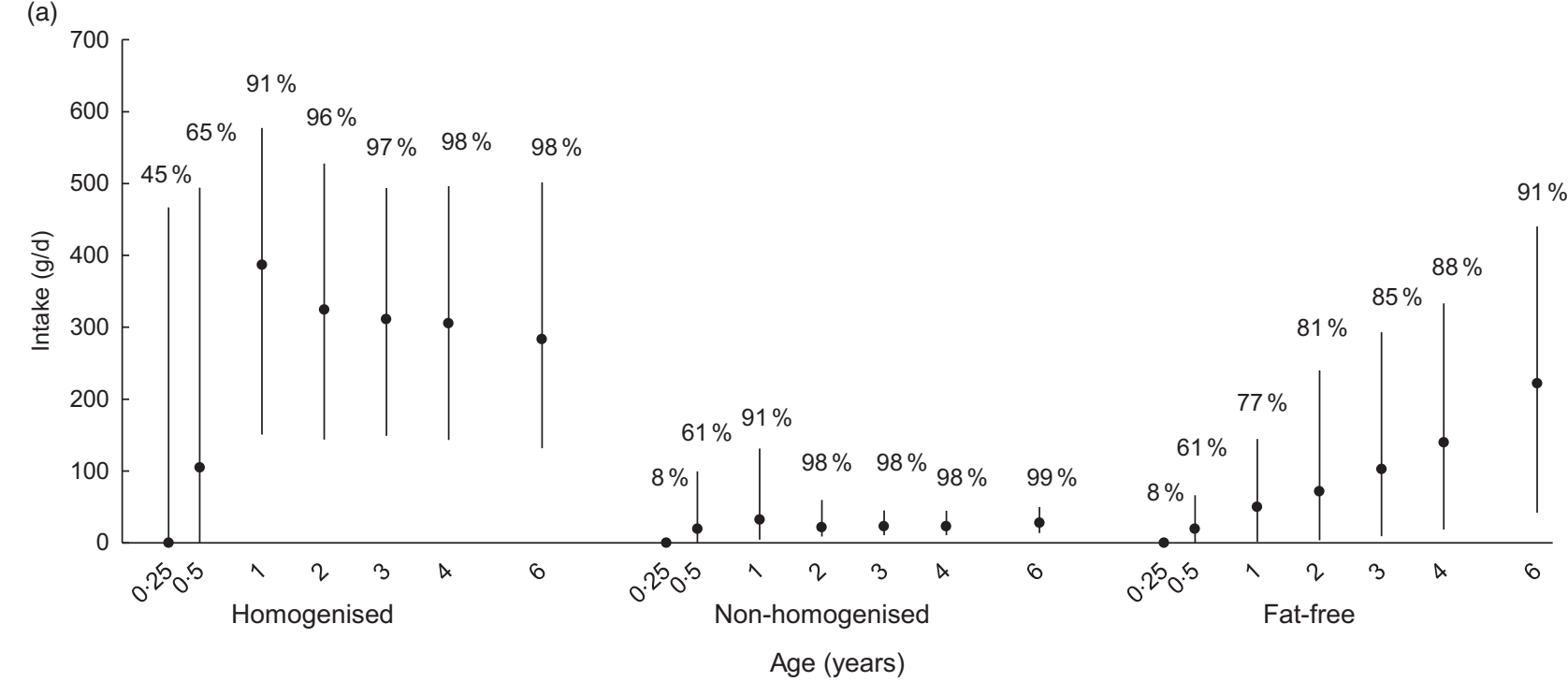

(b)

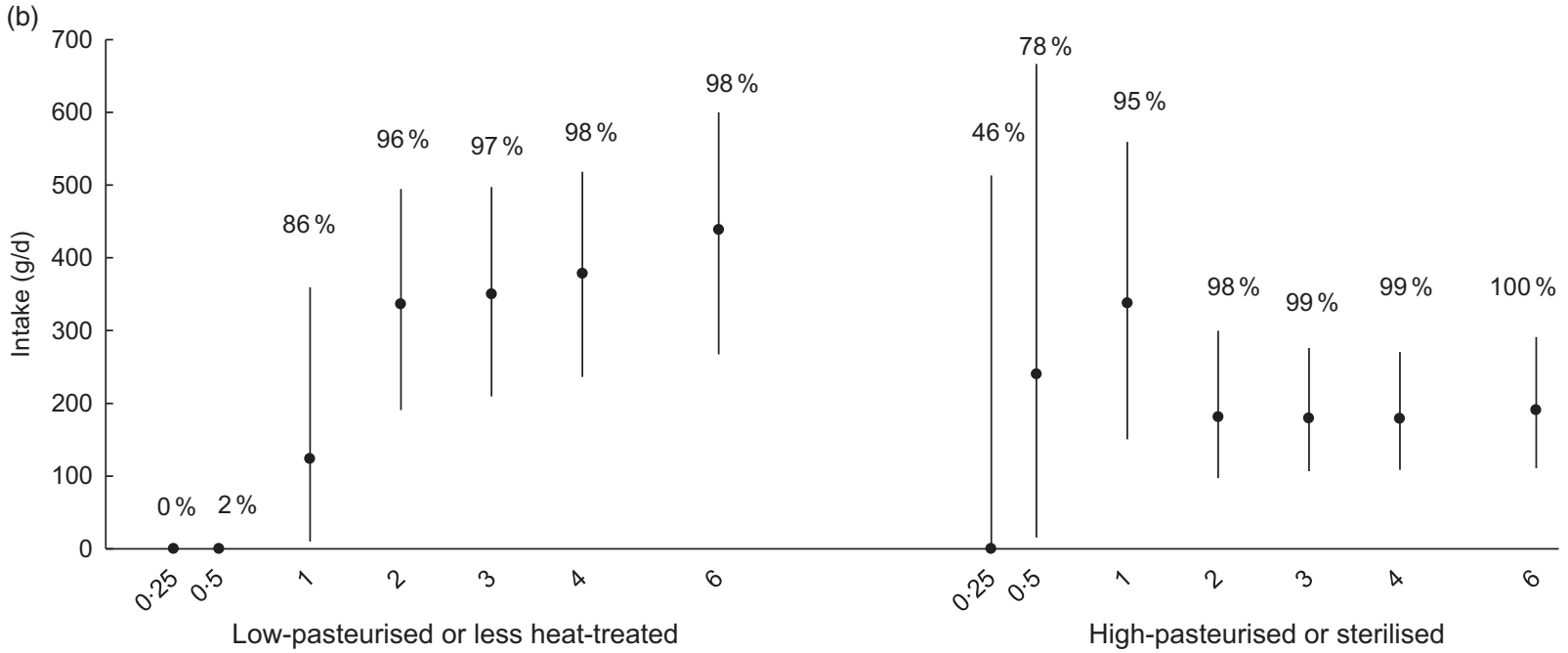

Age (years)

Fig. 2. Consumption of different cows' milk products at the age of 3 months ( $n 5175), 6$ months ( $n 4838), 1$ year $(n 4333), 2$ years $(n 3555)$, 3 years $(n 3330), 4$ years ( $n$ 2979) and 6 years ( $n$ 2267) using classification based on (a) homogenisation; (b) heat treatment. Values are medians, with interquartile ranges represented by vertical bars. Values are calculated based on all the available data for the 5545 children included in the analyses. The percentages above the lines present the proportion of children using the particular milk product at that age point.

The amount of breast milk consumed or energy intake was not associated with the risk of islet autoimmunity. The Cox regression model showed that the cumulative amount of cows' milk products or breast milk up to the age of 6 years was not associated with the islet autoimmunity risk beyond the age of 6 years (online Supplementary Table 1).

\section{Discussion}

In this study, the consumption of cows' milk products was associated with an increased risk of islet autoimmunity (Table 2). This result supports previous observations of the possible role of cows' milk in the development of type 1 diabetes $^{(2-6)}$.

A major strength of this study is the large, longitudinal food record data combined with a frequently updated national food composition database. Using food records instead of FFQ enabled the use of the milk processing classes, which provide us a completely novel exposure assessment. Another advantage of the present study is that we could apply joint model on these data. Joint models are computationally burdensome, but they can take intra-individual variation of the food records into account and are capable of handling incomplete food record data better than the commonly used Cox regression model ${ }^{(21)}$.

The major limitation of this study is that the milk product classification is not unambiguous. The type of a milk product is strongly linked to how it is processed: the milk product type somewhat determines its processing classification ${ }^{(17)}$. Also, the children in the present study carry genetic risk for type 1 diabetes and whether the results can be generalised to the non-risk children is unclear. It should also be considered that the dropout rates at the ages of 6 and 15 years were rather high. Our analysis 
Table 2. Risk of islet autoimmunity (cases $n 246$, total $n 5545$ ) by the age of 6 years associated with breast milk and consumption of different types of milk products based on the basic joint model

(Hazard ratios (HR) and $95 \%$ confidence intervals)

\begin{tabular}{|c|c|c|c|c|c|c|c|c|c|}
\hline & \multicolumn{3}{|c|}{ Unadjusted model* } & \multicolumn{3}{|c|}{ Adjusted model* ${ }^{*}$} & \multicolumn{3}{|c|}{ Energy-adjusted modelł } \\
\hline & $\mathrm{HR}$ & $95 \% \mathrm{Cl}$ & $P$ & $\mathrm{HR}$ & $95 \% \mathrm{Cl}$ & $P$ & $\mathrm{HR}$ & $95 \% \mathrm{Cl}$ & $P$ \\
\hline $\begin{array}{l}\text { Amount of breast milk§ } \\
\text { Conventional classification }\end{array}$ & 0.96 & $0.87,1.05$ & 0.328 & 0.96 & $0.87,1.05$ & 0.357 & 0.96 & $0.90,1.01$ & 0.130 \\
\hline Cows' milk products & 1.07 & $1 \cdot 01,1 \cdot 14$ & 0.019 & 1.06 & $1.00,1 \cdot 13$ & 0.037 & 1.06 & $1 \cdot 02,1 \cdot 11$ & 0.003 \\
\hline Non-fermented milk products & 1.06 & $1.00,1 \cdot 13$ & 0.045 & 1.06 & $1 \cdot 00,1 \cdot 12$ & 0.071 & 1.06 & $1 \cdot 01,1 \cdot 10$ & 0.011 \\
\hline Milk-based infant formulas\|ll & 1.04 & $0.89,1 \cdot 20$ & 0.645 & 1.02 & $0.88,1 \cdot 19$ & 0.767 & 1.01 & $0.89,1.14$ & 0.866 \\
\hline Fermented milk products $\emptyset$ & 1.33 & $1.05,1.70$ & 0.018 & 1.29 & $1.02,1.65$ & 0.038 & 1.35 & $1.10,1.67$ & 0.005 \\
\hline Cheeses & 1.62 & $0.32,8.33$ & 0.561 & 1.57 & $0.30,8.23$ & 0.594 & 1.67 & $0.29,9.80$ & 0.568 \\
\hline \multirow{2}{*}{\multicolumn{10}{|c|}{$\begin{array}{l}\text { Processing-based classification } \\
\text { Homogenisation }\end{array}$}} \\
\hline & & & & & & & & & \\
\hline Homogenised milk products & 1.08 & $1.02,1 \cdot 15$ & 0.007 & 1.08 & $1 \cdot 02,1 \cdot 14$ & 0.010 & 1.07 & $1.03,1.12$ & $<0.001$ \\
\hline Non-homogenised milk products & 1.08 & $0.93,1.26$ & 0.327 & 1.08 & $0.93,1.27$ & 0.324 & 1.06 & $0.93,1.22$ & 0.395 \\
\hline Fat-free milk products & 0.97 & $0.87,1.07$ & 0.482 & 0.96 & $0.87,1.06$ & 0.408 & 0.97 & $0.87,1.07$ & 0.494 \\
\hline \multicolumn{10}{|l|}{ Heat treatment } \\
\hline Low-pasteurised or less heat-treated milk products & 1.03 & $0.96,1.11$ & 0.392 & 1.03 & $0.96,1.11$ & 0.398 & 1.04 & $0.98,1.11$ & 0.210 \\
\hline High-pasteurised or sterilised milk products & 1.07 & $1.00,1.15$ & 0.044 & 1.06 & $0.99,1.14$ & 0.084 & 1.04 & $0.99,1.09$ & 0.105 \\
\hline
\end{tabular}

${ }^{*} \mathrm{HR}$ and $\mathrm{Cl}$ are presented per consumption of $100 \mathrm{~g}$ of food item.

† Adjusted for child's sex, genetic risk and first-degree familial diabetes.

$\ddagger \mathrm{HR}$ and $\mathrm{Cl}$ are presented per consumption of $20 \mathrm{~g} / \mathrm{MJ}$ of food item.

$\S$ For breast milk analysis, cases $n 240$, total $n 5400$.

II Does not include extensively hydrolysed formulas.

I| Does not include cheeses. Cheeses are presented separately.

methodology permitted the inclusion of all the children in the analyses, also those who dropped out before the end of their follow-up. As the joint model remains valid under a broad class of missingness patterns ${ }^{(20)}$, the effect of the dropout rates on the results should not be substantial.

Considering that the children consumed almost half a kg of cows' milk products per $\mathrm{d}$, the risk increment of $7 \%$ per $100 \mathrm{~g}$ of cows' milk products is notable. In contrast to earlier findings from a nested case-control study ${ }^{(4)}$ based on parts of the data of the present one, the present study found that also a higher consumption of fermented milk products was associated with a higher risk of islet autoimmunity. In the present study, there are more cases and longer follow-up than in the previous one and the classification of milk products has been specified ${ }^{(17)}$.

The consumption of infant formulas was not associated with the islet autoimmunity risk. This is in line with a previous observation that the amount of cows' milk consumed at the ages of 3 and 6 months (thus mostly infant formula) was not associated with the risk of islet autoimmunity ${ }^{(24)}$. However, in that previous study, infants' higher cows' milk consumption was associated with a higher risk of primary insulin autoimmunity, which may indicate that risk factors could vary depending on the type of islet autoimmunity. Due to the uses of infant formulas, consumption of strongly heated milk products is high in the first months of life (Fig. 2). However, the consumption of high-pasteurised or sterilised milk products was associated with an increased islet autoimmunity risk in the unadjusted model, unlike infant formulas. The composition of infant formula differs from standard milk - they, for example, contain more lactose and less protein than milk, and the protein fractions are modified. They also have different fat composition with generally added vegetable oils.

Consumption of homogenised milk products was directly associated with the islet autoimmunity risk. The consumption of non-homogenised milk products showed similar but non-significant hazard ratios. Stratification of the milk consumption according to milk processing (Fig. 2) apparently reduced the number of individuals using less commonly consumed food items, which could have compromised statistical power in those comparisons, such as non-homogenised milk products. Therefore, the lack of associations in these should not be considered conclusive. On the other hand, in this study, consumption of fat-free milk products showed no association with the risk of islet autoimmunity, while milk fat has been associated with an increased islet autoimmunity risk previously ${ }^{(4)}$. Altogether, these results indicate that expression of fat in milk may contribute its possible diabetogenic properties. During homogenisation, milk fat globules are broken into smaller particles using pressure. The milk fat globule membrane cannot cover the new droplets completely, and mainly casein micelles are adsorbed on the fat globule surface ${ }^{(25)}$. Also, homogenisation has been shown to reduce the concentration of native proteins in milk $^{(26)}$. In rats, it has been observed that exposure to homogenised milk is associated with higher concentrations of milk protein antibodies compared with non-homogenised milk suggesting different immunisation responses ${ }^{(27)}$, but in humans evidence is lacking.

Several studies have reported a protective association between total or exclusive breast-feeding and the risk of type 1 diabetes ${ }^{(8)}$. We did not see this association in the present study. Previously high intake of breast milk has been associated with a decreased risk for primary insulin autoimmunity only $^{(24)}$.

The cumulative consumption of milk products up to the age of 6 years was not associated with the islet autoimmunity development after the age of 6 years, even though there was a direct association between milk product consumption and the islet autoimmunity risk until the age of 6 years. This indicates that a continuous trigger is needed for the islet autoimmunity to develop, rather than that there would be programming by early milk product consumption. 
This study adds new information on top of the previous evidence on the association between high cows' milk consumption and development of type 1 diabetes. However, as the findings regarding early cows' milk consumption are contrasting, more studies in different study settings should be conducted before changes in nutritional recommendations should be made. As nutrition recommendations are most likely best transferred to the public when they are coherent and enough generic, creating unnecessary restrictions should be avoided. Nationwide, milk and milk products are an important source of several nutrients, for example, $\mathrm{Ca}$, vitamin $\mathrm{D}$, vitamin $\mathrm{B}_{12}$ and iodine ${ }^{(28)}$. Future research should also target identifying the possible components in cows' milk responsible for the associations.

In conclusion, the results of this study are parallel with several previous findings confirming the association between high consumption of cows' milk products and increased risk of islet autoimmunity. The early milk consumption was no longer associated with increased risk of islet autoimmunity onset beyond 6 years. The study did not identify any specific type of milk processing that would clearly stand out as a sole risk factor apart from other milk products. However, the study implicates that future research focusing on the possible association between food consumption and type 1 diabetes should also consider food processing.

\section{Acknowledgements}

We express our gratitude to the children and parents who participated. We thank the DIPP research nurses, doctors, nutritionists, laboratory and food database staff in the University Hospitals of Oulu and Tampere, Faculty of Social Sciences/ Health Sciences of the Tampere University and the Public Health Promotion Unit at the Finnish Institute for Health and Welfare for excellent collaboration over the years.

This work was supported by the Academy of Finland (63672, 68292, 79685, 79686, 80846, 114666, 126813, 129492, 139391, 201988, 210632, 276475 and 308066); European Foundation for the Study of Diabetes (EFSD/JDRF/Lilly Programme); the Juho Vainio Foundation; the Finnish Cultural Foundation; the Future Fund of the University of Helsinki; the Finnish Food Research Foundation; the Competitive State Research Financing of the Expert Responsibility area of Tampere, Turku and Oulu University Hospitals (9E082, 9F089, 9G087, 9H092, 9J147, 9K149, 9L042, 9L117, 9M036, 9M114, 9N086, 9P057, 9R055, 9S074 and 9T072); the JDRF (4-1998-274, 4-1999-731 and 4-2001-435); the European Union (BMH4-CT98-3314); the Novo Nordisk Foundation; the Academy of Finland (Centre of Excellence in Molecular Systems Immunology and Physiology Research 2012-2017, Decision No. 250114) and the Sigrid Jusélius Foundation. The study sponsors had no role in the design, analysis or writing of this article.

K. K., E. S., S. N., J. P., J. N., T. A. and S. M. V. were responsible for the current study design and participated in data interpretation. J. I., J. T., R. V. and M. K. are responsible for the DIPP Study, and S. M. V. for the Nutrition Study within DIPP. K. K. wrote the first version of the manuscript. J. P. and J. N. supervised the planning and the conduct of the data analysis. E. S. performed the data analyses and wrote the statistical methods part. H.-M. T., M. A. and T. E. K. handled the food consumption data processing. S. A. supervised the collection, processing and analysis of the dietary data. J. T., R. V. and M. K. provided the clinical data used in this study and supervised the clinical work. R. V. and M. K. were responsible for the autoantibody analyses and J. I. for the genetic analyses. All the authors reviewed the manuscript. K. K. and E. S. contributed equally to this manuscript.

The authors declare that there are no conflicts of interest.

\section{Supplementary material}

For supplementary material referred to in this article, please visit https://doi.org/10.1017/S0007114520000744

\section{References}

1. Patterson CC, Harjutsalo V, Rosenbauer J, et al. (2019) Trends and cyclical variation in the incidence of childhood type 1 diabetes in 26 European centres in the 25 year period 1989-2013: a multicentre prospective registration study. Diabetologia $\mathbf{6 2}$, 408-417.

2. Virtanen SM, Hyppönen E, Läärä E, et al. (1998) Cow's milk consumption, disease-associated autoantibodies and type 1 diabetes mellitus: a follow-up study in siblings of diabetic children. Childhood Diabetes in Finland Study Group. Diabet Med 15, 730-738.

3. Virtanen SM, Läärä E, Hyppönen E, et al. (2000) Cow's milk consumption, HLA-DQB1 genotype, and type 1 diabetes: a nested case-control study of siblings of children with diabetes. Childhood Diabetes in Finland Study Group. Diabetes 49, 912-917.

4. Virtanen SM, Nevalainen J, Kronberg-Kippilä C, et al. (2012) Food consumption and advanced $ß$ cell autoimmunity in young children with HLA-conferred susceptibility to type 1 diabetes: a nested case-control design. Am J Clin Nutr 95, 471-478.

5. Wahlberg J, Fredriksson J, Nikolic E, et al. (2005) Environmental factors related to the induction of beta-cell autoantibodies in 1-yr-old healthy children. Pediatr Diabetes 6, 199-205.

6. Lamb MM, Miller M, Seifert JA, et al. (2015) The effect of childhood cow's milk intake and HLA-DR genotype on risk of islet autoimmunity and type 1 diabetes: the Diabetes Autoimmunity Study in the Young. Pediatr Diabetes 16, 31-38.

7. Virtanen S (2016) Dietary factors in the development of type 1 diabetes. Pediatr Diabetes 17, 49-55.

8. Patelarou E, Girvalaki C, Brokalaki H, et al (2012) Current evidence on the associations of breastfeeding, infant formula, and cow's milk introduction with type 1 diabetes mellitus: a systematic review. Nutr Rev 70, 509-519.

9. Vaarala O, Ilonen J, Ruohtula T, et al. (2012) Removal of bovine insulin from cow's milk formula and early initiation of beta-cell autoimmunity in the FINDIA pilot study. Arch Pediatr Adolesc Med 166, 608-614.

10. Knip M, Åkerblom HK, Altaji E, et al. (2018) Effect of hydrolyzed infant formula vs conventional formula on risk of type 1 diabetes the TRIGR randomized clinical trial. JAMA $\mathbf{3 1 9}$, $38-48$.

11. Knip M, Virtanen SM, Seppä K, et al. (2010) Dietary intervention in infancy and later signs of beta-cell autoimmunity. $N$ Engl J Med 363, 1900-1908. 
12. Ollikainen P \& Muuronen K (2013) Determination of insulin-like growth factor-1 and bovine insulin in raw milk and its casein and whey fractions after microfiltration and ultrafiltration. Int Dairy J 28, 83-87.

13. Qi PX, Ren D, Xiao Y, et al. (2015) Effect of homogenization and pasteurization on the structure and stability of whey protein in milk. J Dairy Sci 98, 2884-2897.

14. Bogahawaththa D, Buckow R, Chandrapala J, et al. (2018) Comparison between thermal pasteurization and high pressure processing of bovine skim milk in relation to denaturation and immunogenicity of native milk proteins. Innov Food Sci Emerg Technol 47, 301-308.

15. Kupila A, Muona P, Simell T, et al. (2001) Feasibility of genetic and immunological prediction of type I diabetes in a population-based birth cohort. Diabetologia 44, 290-297.

16. Schoen S, Sichert-Hellert W \& Kersting M (2009) Validation of energy requirement equations for estimation of breast milk consumption in infants. Public Health Nutr 12, 2309-2316.

17. Koivusaari K, Niinistö S, Takkinen H, et al. (2018) A novel processing-based classification and conventional food grouping to estimate milk product consumption in Finnish children. Int Dairy J 86, 96-102.

18. Kukko M, Kimpimäki T, Korhonen S, et al. (2005) Dynamics of diabetes-associated autoantibodies in young children with human leukocyte antigen-conferred risk of type 1 diabetes recruited from the general population. J Clin Endocrinol Metab 90, 2712-2717.

19. Siljander HT, Simell S, Hekkala A, et al. (2009) Predictive characteristics of diabetes-associated autoantibodies among children with HLA-conferred disease susceptibility in the general population. Diabetes 58, 2835-2842.

20. Rizopoulos D (2012) Joint Models for Longitudinal and Time-to-Event Data. New York: Chapman and Hall/CRC.

21. Syrjälä E, Nevalainen J, Peltonen J, et al. (2019) A joint modeling approach for childhood meat, fish and egg consumption and the risk of advanced islet autoimmunity. Sci Rep $\mathbf{9}, 7760$.

22. Willett WC, Howe GR \& Kushi LH (1997) Adjustment for total energy intake in epidemiologic studies. Am J Clin Nutr $\mathbf{6 5}$, $1220-1231 S$.

23. Rizopoulos D (2010) JM: an R Package for the joint modelling of longitudinal and time-to-event data. J Stat Softw 35, 1-33.

24. Niinistö S, Takkinen HM, Erlund I, et al. (2017) Fatty acid status in infancy is associated with the risk of type 1 diabetes -associated autoimmunity. Diabetologia 60, 1223-1233.

25. Michalski MC \& Januel C (2006) Does homogenization affect the human health properties of cow's milk?. Trends Food Sci Technol 17, 423-437.

26. Brick T, Ege M, Boeren S, et al. (2017) Effect of processing intensity on immunologically active bovine milk serum proteins. Nutrients 9, E963.

27. Feng CG \& Collins AM (1999) Pasteurisation and homogenisation of milk enhances the immunogenicity of milk plasma proteins in a rat model. Food Agric Immunol 11, 251-258.

28. Valsta L, Kaartinen N, Tapanainen H, et al. (2018) Ravitsemus Suomessa - FinRavinto 2017-tutkimus (Nutrition in FinlandThe National FinDiet 2017 Survey). Institute for Health and Welfare (THL). Report 12/2018. Helsinki, Finland. 